\title{
Endothelial cells instruct macrophages on how to Rspond to lung injury
}

\author{
The lung endothelial cell-derived angiocrine Rspondin3 activates Wnt- $\beta$-catenin signaling in interstitial \\ macrophages, leading to a metabolic-epigenetic reprogramming of interstitial macrophages that drives \\ anti-inflammatory responses and attenuates endotoxin-induced lung injury.
}

\section{Thomas Marichal}

i ike the skin and the gut, mammalian lungs are at the interface between the host and the external world and are continuously exposed to microorganisms, microbial products, allergens and other noxious stimuli. To allow efficient gas diffusion and to support life, the air-blood barrier must be very thin, and it must be tightly regulated to avoid excessive inflammatory damage. In the current issue of Nature Immunology, Zhou et al. ${ }^{1}$ have identified an anti-inflammatory angiocrinemacrophage axis that contributes to the attenuation of lung inflammatory injury in endotoxemic mice.

Using multiple strains of genetically modified mice in combination with ex vivo bone-marrow-derived macrophage (BMDM) stimulation experiments, Zhou et al. provide convincing experimental evidence that the endothelial cell (EC)-derived angiocrine Rspondin 3 is triggered by lipopolysaccharide (LPS) and drives the expansion of anti-inflammatory and tissue-protective interstitial macrophages (IMs) through macrophage-intrinsic LGR4- $\beta$-catenin-mediated metabolicepigenetic reprogramming (Fig. 1a).

Macrophages are present in most mammalian tissues and exhibit remarkable microenvironment-driven heterogeneity and plasticity to fulfill tissue-specific and context-specific functions ${ }^{2,3}$. Besides the well-known alveolar macrophages (AMs), the steady-state lung is populated by parenchymal IMs made up of distinct subpopulations in specific niches ${ }^{4-6}$. During inflammation, infection or tissue damage, monocytes can enter the tissue, be imprinted by stress and microenvironmental signals provided by the local niche and undergo differentiation into IMs that are thought to regulate immune, inflammatory and tissue repair responses ${ }^{7,8}$. Yet the identification of niche-derived signals imprinting lung IMs during homeostasis and inflammation, as well as the IM-intrinsic molecular pathways involved, remain largely unknown.

a

Wild-type mice or mice with EC-intrinsic Rspo3 deficiency treated with Rspondin3 i.v.

b Mice with EC-intrinsic Rspo3 deficiency or mice with macrophage-intrinsic Lgr4, Ctnnb or Tet2 deficiency
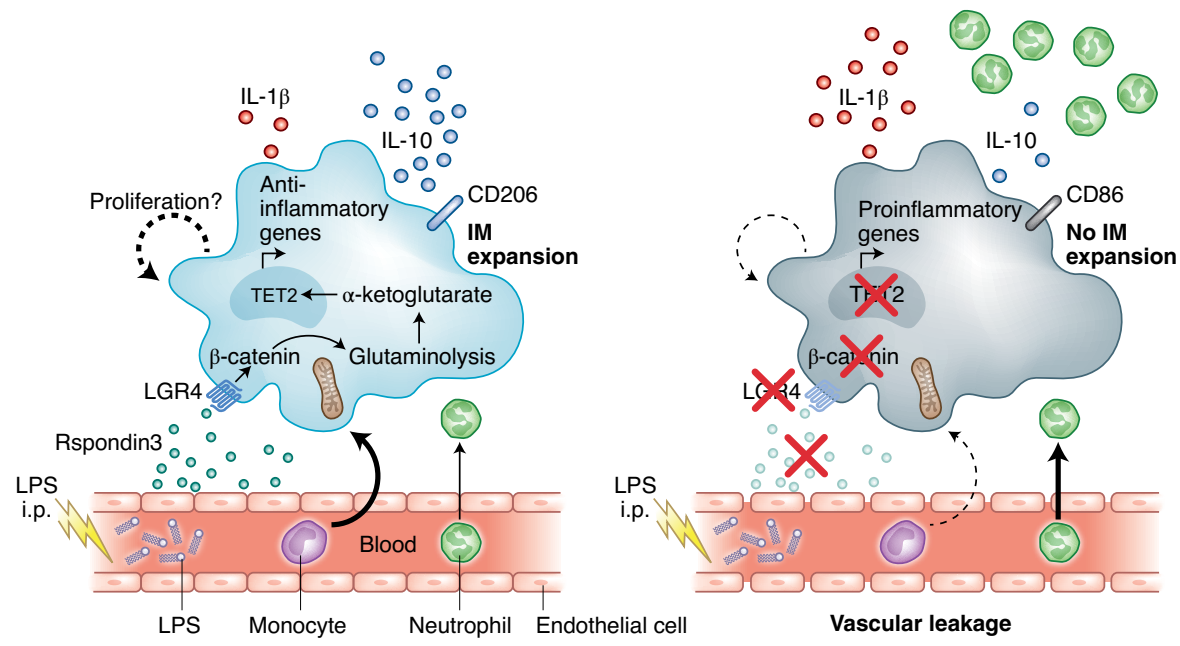

Fig. 1| A new angiocrine-macrophage axis negatively regulates lung inflammatory injury in endotoxemic mice. a, In wild-type mice, the LPS-triggered release of Rspondin3 (Rspo3) by lung ECs activates LGR4- $\beta$-catenin signaling in IMs, leading to a metabolic-epigenetic reprogramming of IMs that drives their expansion and anti-inflammatory responses, including expression of anti-inflammatory surface markers, such as CD206, and elevated levels of IL-10. The metabolic-epigenetic pathway involves glutaminolysis to generate $\alpha$-ketoglutarate, which serves as a cofactor for TET2 methylcytosine dioxygenase, which in turn catalyzes DNA hydroxymethylation and activation of anti-inflammatory genes. Similar observations can be made in LPS-challenged mice with EC-specific deletion of Rspo3 that are supplemented with Rspondin3 i.v. b, In mice conditionally deficient for Rspo3 in ECs or in mice with a macrophage-intrinsic deficiency in Lgr4, Ctnnb or Tet2, i.p. LPS treatment does not lead to the expansion of IMs, which instead exhibit a proinflammatory profile, including high surface expression of CD86 and elevated levels of IL-1 $1 \beta$. This is associated with exacerbated features of lung inflammatory injury, such as elevated neutrophil activity and counts and vascular leakage. Other immune cells are not depicted for clarity.

Since lung ECs are located in proximity to extravasating monocytes and $\mathrm{IMs}^{4,5}$, they represent a potential cellular source of signals tailoring IM identity. To test this hypothesis, Zhou et al. performed a secretome assay on conditioned medium from LPS-activated mouse lung ECs and found the Wnt signaling activator Rspondin3 to be one of the top secreted proteins. In vitro, the authors first showed that stimulation of BMDMs with conditioned medium from LPS-stimulated mouse lung ECs triggered an anti-inflammatory macrophage phenotype characterized by elevated expression of CD206, CD301, Arginase 1 and interleukin(IL)-10 and reduced expression of the proinflammatory markers CD86, CD80, TNF and iNOS as compared to unstimulated BMDMs. Second, they showed that such antiinflammatory macrophage programming, including the production of IL-10, a 
cytokine important for IM homeostatic functions ${ }^{8}$, was also induced by stimulation of BMDMs with recombinant Rspondin3 in the presence or absence of LPS stimulation. Third, they performed BMDM stimulation experiments using conditioned medium from mouse lung ECs isolated either from wild-type (WT) mice or from mice conditionally deficient in Rspondin 3 in ECs $\left(R s p o 3^{\mathrm{EC}-I_{-}}\right)$that were previously treated intraperitoneally (i.p.) with LPS (endotoxemic mice) to demonstrate that the anti-inflammatory features of BMDMs were dependent on EC-intrinsic Rspondin3 via contact-independent mechanisms.

To gain insight into the relevance of these findings for lung IMs in vivo, the authors performed elaborate lung myeloid cell profiling using mass cytometry in Rspo3 ${ }^{\mathrm{EC}-/-}$ and WT mice at baseline and after i.p. LPS challenge with or without intravenous (i.v.) treatment with Rspondin3. While EC-intrinsic Rspondin3 deficiency did not affect the numbers and the phenotype of IMs at baseline, the expansion of IMs (defined as CD45 ${ }^{+}$Ly-6G/C- ${ }^{-}$D 64 $4^{+}$MerT $\mathrm{K}^{+} \mathrm{CD} 11 \mathrm{~b}^{+}$Siglec- $\mathrm{F}^{-}$cells) and their shift toward an anti-inflammatory phenotype after LPS challenge was entirely absent in Rspo $3^{\mathrm{EC}-/-}$ mice and could only be restored by Rspondin 3 i.v. treatment. Instead, IMs displayed a proinflammatory phenotype after LPS treatment in Rspo $3^{\mathrm{EC}-1-}$ mice, which was associated with exacerbated signs of LPS-induced acute lung injury, including increased lung neutrophil counts and higher vascular permeability, as well as lower survival as compared to WT mice (Fig. 1b). Of note, the abnormalities observed in endotoxemic Rspo $3^{\mathrm{EC}-/-}$ mice could be restored by Rspondin 3 i.v. treatment. The expansion and the anti-inflammatory phenotype of IMs was also shown to be dependent on EC-intrinsic Rspondin3 five days after intratracheal bleomycin treatment, a model in which Lyve $1^{+}$IMs have previously been shown to restrain inflammation and fibrosis ${ }^{4}$. Together, these data suggest that lung ECs release Rspondin3 in response to lung-damaging stimuli to imprint IMs with an anti-inflammatory identity that mitigates the extent of lung injury (Fig. 1a).

Next, the authors investigated the intrinsic molecular players involved in Rspondin3-mediated imprinting of IMs and focused their attention on the contributions of LGR4, highly and specifically expressed by IMs, and the Wnt $-\beta$-catenin signaling pathway, activated in IMs after i.v. administration of Rspondin3. Using BMDMs deficient in Lgr4 or Ctnnb (encoding $\beta$-catenin) and mice conditionally deficient in Lgr4 or Ctnnb in macrophages, the authors accumulated experimental evidence showing LGR4 expression and the activation of the $\beta$-catenin pathway in IMs were mediating both Rspondin3-induced programming of IMs toward an anti-inflammatory phenotype and the attenuation of LPS-induced inflammatory lung injury (Fig. 1b). These results are concordant with a previous report showing that germline Lgr4-deficient mice were highly susceptible to endotoxemia and exhibited increased production of proinflammatory cytokines by macrophages ${ }^{9}$. Nevertheless, the role of LGR4 in macrophages seems to be context-specific, as it has recently been suggested that LGR4 promotes a pro-inflammatory macrophage program in a model of myocardial infarction ${ }^{10}$.

Metabolic adaptations and epigenetic modifications are increasingly recognized as contributing to macrophage diversity ${ }^{11,12}$. In the last part of the study, Zhou et al. analyzed BMDMs in vitro and found that Rspondin-3 promoted oxidative phosphorylation via glutaminolysis and the generation of $\alpha$-ketoglutarate in an LGR4- and $\beta$-catenin-dependent manner. Furthermore, Rspondin-3 also increased the activity of TET methylcytosine dioxygenase, which utilizes $\alpha$-ketoglutarate as a cofactor, to catalyze DNA hydroxymethylation and activate anti-inflammatory genes, highlighting an Rspondin-3-induced metabolic-epigenetic anti-inflammatory reprogramming in BMDMs. Interestingly, the authors found that mice conditionally deficient in Tet 2 in macrophages exhibited the same deficit in the expansion of anti-inflammatory IMs and in the attenuation of inflammatory lung injury as that observed in Rspo $3^{\mathrm{EC}-/-}$ mice and in mice conditionally deficient in $L g r 4$ or Ctnnb in macrophages, suggesting that TET2-mediated epigenetic reprogramming also occurs in IMs in vivo after systemic LPS challenge (Fig. 1b).

The work of Zhou et al. makes important contributions to our understanding of the niche occupied by and the identity of lung IMs during inflammation by identifying an angiocrine-macrophage axis that shapes the anti-inflammatory phenotype of IMs and attenuates lung inflammatory injury. It would be interesting to extend such investigations to other organs wherein IMs are present, for example, the fat, the heart or the dermis ${ }^{4}$, and to assess whether the Rspondin3-LGR4-Wnt- $\beta$-catenin pathway can also reprogram such IMs toward an anti-inflammatory phenotype to regulate excessive inflammation elsewhere in the body.

At baseline, the identity of IMs as assessed in this study was not significantly affected by Rspondin 3 treatment, by EC-intrinsic Rspo3 deficiency nor by macrophage-specific Lgr4, Ctnnb or Tet2 deficiency. There is therefore no experimental evidence at this point to support that endothelial-derived Rspondin 3 and the corresponding pathway in IMs are involved in the maintenance of steady-state IMs. Additional investigations looking at the abundance, phenotype, secretory profile and single-cell transcriptome of previously identified steady-state IMs subpopulations ${ }^{4-6}$ in those transgenic animals at baseline may reveal that ECs also release signals imprinting the identity of IM subpopulations during homeostasis. The identification of blood-vessel-associated $\mathrm{IMs}^{4}$ and nerve-associated $\mathrm{IMs}^{4,6}$ in lungs and across tissues is consistent with the idea that both ECs and nerves release signals that instruct IM subpopulations at baseline.

The relevance of these findings for human diseases is currently unknown. Nevertheless, in the long term, identifying the niche cells and the signals that are important to control the beneficial, anti-inflammatory functions of lung IMs might open new therapeutic avenues for diseases in which dysregulation of lung macrophages has been implicated, such as the most severe forms of COVID-19 and other acute respiratory distress syndromes ${ }^{13}$.

\section{Thomas Marichal (D) 1,2,3凶 \\ ${ }^{1}$ Laboratory of Immunophysiology, GIGA Institute, Liège University, Liege, Belgium. ${ }^{2}$ Faculty of Veterinary Medicine, Liège University, Liege, Belgium. ${ }^{3}$ WELBIO, Walloon Excellence in Life Sciences and Biotechnology, Wallonia, Belgium.

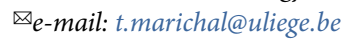

Published online: 2 October 2020 https://doi.org/10.1038/s41590-020-00806-z

References

1. Zhou, B. et al. Nat. Immunol. https://doi.org/10.1038/s41590-0200764-8 (2020).

2. Blériot, C., Chakarov, S. \& Ginhoux, F. Immunity 52, 957-970 (2020)

3. Guilliams, M., Thierry, G. R., Bonnardel, J. \& Bajenoff, M. Immunity 52, 434-451 (2020).

4. Chakarov, S. et al. Science 363, eaau0964 (2019).

5. Schyns, J. et al. Nat. Commun. 10, 3964 (2019).

6. Ural, B. B. et al. Sci. Immunol. 5, eaax8756 (2020).

7. Ginhoux, F., Schultze, J. L., Murray, P. J., Ochando, J. \& Biswas, S. K. Nat. Immunol. 17, 34-40 (2016).

8. Schyns, J., Bureau, F. \& Marichal, T. J. Immunol. Res. 2018, 5160794 (2018).

9. Du, B. et al. J. Biol. Chem. 288, 15131-15141 (2013).

10. Huang, C.-K. et al. Circ. Res. https://doi.org/10.1161/ CIRCRESAHA.119.315807 (2020).

11. Artyomov, M., Sergushichev, A. \& Schilling, J. D. Semin. Immunol. 28, 417-424 (2016).

12. Saeed, S. et al. Science 345, 1251086 (2014).

13. Merad, M. \& Martin, J. C. Nat. Rev. Immunol. 20, 355-362 (2020).

Competing interests

The author declares no competing interests. 